\title{
INTEGRATED METHODOLOGY FOR THE MANAGEMENT OF HUMAN EXPOSURE TO AIR POLLUTANTS
}

\author{
MARCO SCHIAVON ${ }^{1}$, ELENA CRISTINA RADA ${ }^{2}$, LUCA ADAMI $^{1}$, \\ FEDERICA FOX ${ }^{1} \&$ MARCO RAGAZZI $^{1}$ \\ ${ }^{1}$ Department of Civil, Environmental and Mechanical Engineering, University of Trento, Italy \\ ${ }^{2}$ Department of Theoretical and Applied Sciences, University of Insubria, Italy
}

\begin{abstract}
The assessment of the impacts of future civil/industrial plants on air quality is traditionally based on the environmental assessment procedure, which, however, may lead to different conclusions depending on the choice of the target area and the emission sources already present, and may underestimate the global impacts on health, society and economy. The paper proposes an organic multi-step methodology to carry out health risk assessments on a target area, to assess the incidence of emissive activities under evaluation for authorisation, and, as an additional matter of novelty, to study mitigation/compensation measures to take their future impacts back to acceptable levels, if their contributions are expected to exceed acceptable risk parameters. Criteria for the definition of target areas, emission calculation, dispersion modelling, the health risk assessment procedure, the verification of the risk acceptability and the compensation approach are described in detail. The current potential limitations of the approach are discussed and solutions to overcome such weak points are proposed. In view of a sustainable integration of new activities within local contexts, this methodology aims at guiding decision makers to carry out the screening of projects in terms of health impacts, according to a standardised approach.

Keywords: dispersion modelling, health risk, emissions, air quality, cancer risk, hazard index, environmental impact, external costs, environmental sustainability.
\end{abstract}

\section{INTRODUCTION}

Air pollution is the first environmental cause of worsening of human health and/or mortality on Earth, causing about 7 million deaths per year [1]-[4]. In the 28 countries of the European Union, following the exposure to particulate matter (PM), ozone $\left(\mathrm{O}_{3}\right)$ and nitrogen dioxide $\left(\mathrm{NO}_{2}\right)$, air pollution is believed to be responsible for the death of 483,400 people per year [5]. Among other environmental issues, the traditional environmental impact assessment procedure evaluates the compatibility of future activities with the air quality of the area where those would be located and the compliance with health risk parameters of acceptability. Indeed, health protection should be the ultimate purpose of the authorisation procedure for environmental impact assessment. However, the conventional approach presents some limitations [4], [6]-[10]:

1. the health risk may significantly vary within the area of study, depending on the local prevailing mechanisms of dispersion of the air pollutants, on the spatial distribution of the residents and on the other emission sources that may be present in the area;

2. the definition of the study area itself requires a standardised approach, since the impacts of one or more emission sources quantitatively depend on the extension and location of the target area;

3. although a new activity may comply with health risk acceptability parameters, it may also entail increased social costs, since health impacts, though considered as acceptable, might increase;

4. the conventional approach is based on the use of different methodologies, which requires different input data and formats, and does not follow a standard procedure. 
The novelty of this paper consists in the proposal for an organised and organic multi-step methodology that allows local administrators and decision makers to:

1. carry out the assessment of the risk for health related to air pollution in a target area through an integrated approach;

2. evaluate scenarios that consider the presence of additional emissive activities and the acceptability of their supplemental impacts; and

3. consider possible mitigation measures to compensate the authorisation of a new activity, in order to improve (or, at least, to keep as constant) the status of health in the area.

A scheme of the multi-step methodology that will be discussed in the paper is presented in Fig. 1. The health risk assessment is carried out through the traditional methods of air dispersion modelling and the estimation of the intake of contaminants through the relevant routes of exposure, but the procedure is presented in a unified way and with a logical structure, to facilitate the work of local authorities. The evaluation of scenarios including the activity of additional emission sources allows assessing the local incidence of a new planned industrial/civil plant with respect to the current situation. Finally, the compensation approach should be used when the new activity, in spite of adopting the best available techniques, is expected to induce a health risk that exceeds the criteria of acceptability in the target area. The compensation procedure may translate, for instance, into obliging the owners of the new activity to invest in enhanced air pollution control systems for plants that had been already authorised in the area and that have room for improvement in air pollution control or energy saving policies. As another example, in the case of new waste-to-energy plants, the compensation approach may consist in using the electric energy produced by the plant and ask the owners of the plant to install electrical heating systems in dwellings, as integration (partial substitution) of domestic boilers fed by fossil fuels or wood stoves, the latter being known sources of PM [11], [12].

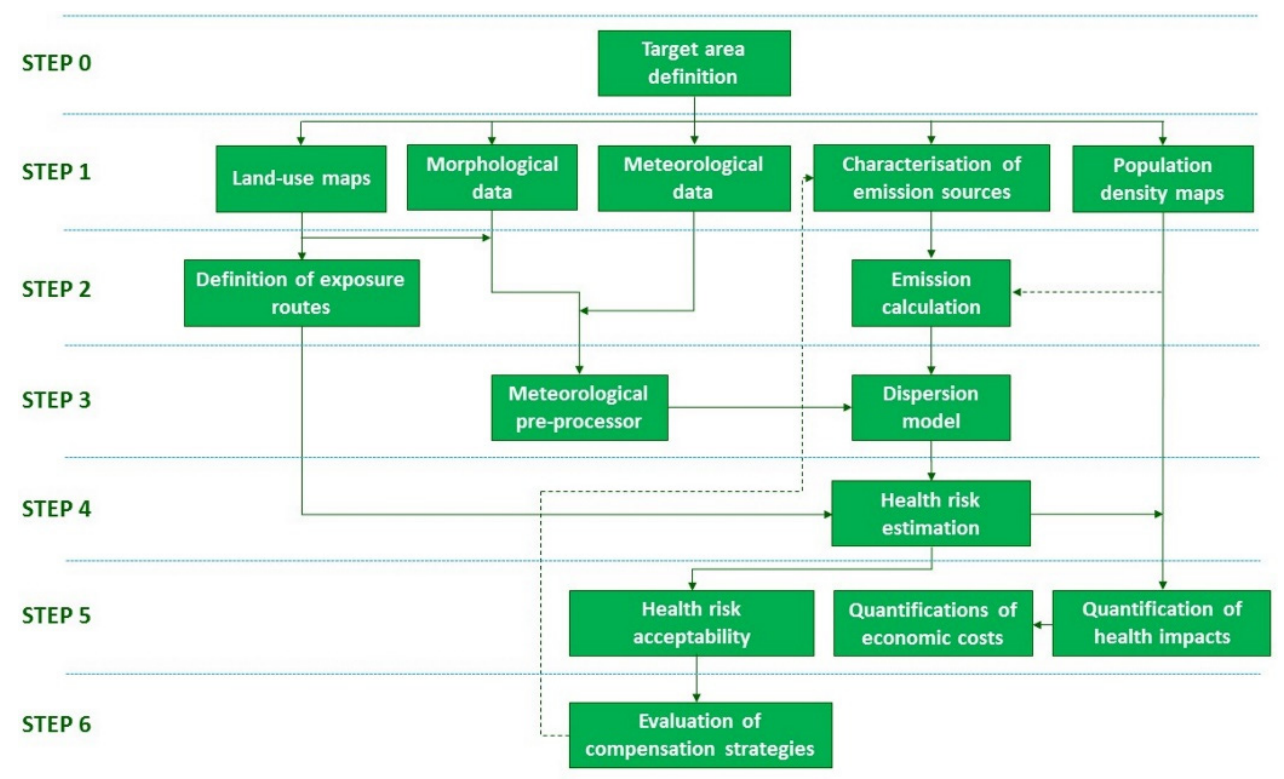

Figure 1: Conceptual scheme of the integrated methodology for the management of human exposure to air pollutants. 


\section{METHODOLOGY}

\subsection{Selection of the target area}

The definition of the target area is a necessary step to identify the dominant routes of exposure, to define the limits of the computation domain of the dispersion model, to estimate the impacts on health, to evaluate the influence of additional emission sources and of possible mitigation strategies. To quantify the impacts, the target area cannot be limited to the area including the industrial sources to investigate, but should be extended to the adjacent municipalities or residential areas, and up to a variable distance from the point of interest that depends on the possible presence of orography, which may impede the dispersion of pollutants. As a reference criterion, the target area should include all the municipalities/ districts contained in a radius of $5 \mathrm{~km}$ from the industrial source (or, if more than one, from the barycentre of the industrial sources). In addition, if important external emission sources are present (e.g., an industrial hub), the target area should include a buffer containing the municipalities/districts adjacent to the area defined by the previous criterion, in order to account for the possible influence of external sources on the air quality of the inner target area. Concerning the horizontal grid resolution of the dispersion model, the higher the resolution, the higher the accuracy of the heath impact estimation. Cells with sides of $100 \mathrm{~m}$ could be a reasonable compromise between accuracy of the results and computational efforts. If the target area includes the buffer, the latter can have a lower horizontal resolution (i.e., higher cell dimensions), which can be imposed with a nesting procedure in the dispersion model.

\subsection{Data collection and emission calculation}

The collection of data is functional to the simulations of the dispersion of air pollutants and to the health risk quantification. The management of data must be carried out on a georeferenced base, i.e., in a geographical information system (GIS) environment.

In addition to direct intake of air pollutants via inhalation, other routes of exposure may be significant in determining the daily dose of air contaminants: the diet, the dermal contact with contaminated food, the dermal contact with soil and the accidental soil ingestion. If the target area includes cultivated lands, pastures and/or places where kids can get into contact with soil (e.g., schools and playgrounds), the air pollutants that deposit to soil may activate those additional exposure routes. Accidental soil ingestion may be a significant route of exposure for children, especially if schools or parks are present in the area [13]. A basic knowledge of the area and the consultation of land-use maps, such those created by the CORINE Land Cover project [14], help identifying the relevant exposure routes to consider. Every land-use class of the CORINE maps also reports a value of the surface roughness, which is an essential parameter to the meteorological pre-processor of the selected dispersion model for the calculation of the wind field in the domain. Depending on the size of the computation domain of the dispersion model, the urban fabric might be explicitly defined rather than parametrising it by defining surface roughness values compatible with the land use [10]. In this case, an elevation map of the buildings must be retrieved or specifically developed.

If orography is present in the target area, a digital elevation model (DEM) must be included in the dispersion model. DEMs are available as georeferenced raster maps on various online databases. The DEM should have a horizontal resolution lower or equal to that of the computation domain of the dispersion model. 
The meteorological pre-processors of dispersion models require hourly meteorological data relative to one or more weather stations in the area or to a regional/global scale model (e.g., WRF). Depending on the dispersion model chosen, the essential data may vary. When the purpose is the assessment of the impacts on health, as in the present case, meteorological data should cover a whole solar year of observations.

It is then essential to retrieve data on the emissions of the largest possible number of pollutants from the largest possible number of sources. In the case of point sources (e.g., chimneys of mills or centralised district heating systems), the data consists in: the location and elevation of the source, the temperature of the gas emitted, the airflow rates, the concentration of pollutants at the stack level, the possible presence of buildings near the source. Those data can be retrieved through the environmental assessment reports. If accurate emission values can be estimated for activities subject to environmental impact assessment, the estimation of the emissions from other sources has a much higher degree of uncertainty. This is the case of road traffic, whose emissions must be estimated based on: a characterisation of the vehicle fleet (usually available from national statistics databases), on the vehicle fluxes along the road network (usually available from local authorities) and on emission factors available in the literature. At a European level, emissions factors for road traffic are provided by the European Environment Agency (EEA) [15] and can be calculated by applying the COPERT 5 algorithm [16]. Roads are usually treated as linear sources in dispersion modelling, thus by approximation to polylines in GIS environments. If aggregate emission factors are available, some activities are conveniently modelled as area sources, due to their ubiquity [17]. This is the case, for instance, of small industries and diffuse sources (e.g., de-centralised domestic heating), which could be approximated to polygons. The emissions from domestic heating could be weighed on the resident population, by retrieving population density maps from regional/national statistics institutions. Regional air pollutant inventory emissions, like the case of INEMAR in Italy [18], turn particularly useful to this purpose. The emissions from other specific areal sources (waste landfills or wastewater treatment plants) may be estimated with the use of national or international databases, like EEA emission inventory [15]. To reduce the uncertainties related to this approximated approach, daily, weekly and yearly patterns of emissions should be assumed. All the mass flow rates of pollutants should cover the same period of meteorological data. Consistently with meteorological data, dispersion models require hourly values of the mass flow rates of the pollutants considered. Given a specific dispersion model, emissions and meteorological data play the most important role in determining the accuracy of the simulation results. Thus, the more emission values are accurate, the higher is the accuracy of the output.

Besides the emission sources located in the target area (with the possible inclusion of the buffer area), it is important to consider the background annual mean concentrations of as more pollutants as possible, by retrieving the data measured by rural background air quality stations within or close to the computation domain. Interpolation methods, like the ordinary Kriging method [19], could be used to obtain more realistic background concentration maps if more than one rural background stations are available.

In addition to weighing the emissions from diffuse sources, population density maps are essential to quantify the impacts in terms of the number of cases of cancer, hospitalisation and mortality expected on an annual basis, and to estimate the economic costs for the society.

\subsection{Dispersion modelling}

The meteorological data, recorded by one or more meteorological stations within the target area or obtained by regional-scale models, are the input data to the meteorological pre- 
processor of the selected dispersion model. Some manipulations are necessary to convert the data into the format required by the pre-processor. Depending on the dispersion model, vertical profiles of wind, temperature and pressure may be requested as input data. The preprocessor computes the horizontal and vertical wind fields that are necessary to calculate the dispersion of air pollutants within the atmosphere. Generally, the output from the dispersion model consists in maps of annual mean and maximum concentrations and, in the case of macropollutants, concentrations compatible with the limit values defined by the environmental legislation. The concentrations simulated for each pollutant must be added to the background concentrations of the same pollutants (if available) measured at rural background sites. In the case that other exposure routes are relevant for the target area, in addition to inhalation, atmospheric depositions should be selected besides air concentrations as the second desired output of the dispersion model.

\subsection{Health risk estimation}

Diet, dermal contact and accidental soil ingestion are relevant pathways for inorganic (heavy metals) and organic (dioxin and polycyclic aromatic hydrocarbons) micropollutants, which tend to accumulate in soil and in vegetable and animal tissues, due to their physical-chemical properties. Such pollutants may of course enter the human body through inhalation too. The estimation of the health risk by exposure to micropollutants is carried on the basis of the guidelines developed by the United States Environmental Protection Agency (US-EPA) for the calculation of the cancer and non-cancer risks [20]. The US-EPA guidelines focus on the long-term effects of micropollutants. All micropollutants may induce toxic non-carcinogenic effects, whereas only some of them are carcinogens: dioxin, polycyclic aromatic hydrocarbons, some volatile organic compounds like benzene and formaldehyde, and some metals including cadmium, arsenic, mercury, nickel and chromium VI [21]. Through specific relations that depend on the exposure route, the guidelines allow estimating the daily intake of each contaminant and the associated risk, by starting from annual mean concentrations and deposition values. The calculation of the exposure through the diet requires considering the statistics on food consumption for the target population, which are usually elaborated by national statistics institutes, like the Italian National Institute for the Research on Food and Nutrition (INRAN) [22]. The estimation of the overall risk is carried out through the superposition principle, thus by assuming that the risk related to a single contaminant can be added to the risks related to the others. By applying the US-EPA guidelines to each cell of the computation domain, it becomes possible to obtain maps of the individual risk (related to the single pollutant) for cancer or non-cancer effects, or a map of the overall (by summing all contributions) cancer risk. As a precautionary measure, the present methodology presented here suggests assuming that all the population living in the target consume only locally-grown food, thus subject to the potential contamination that is the object of the study.

Although the US-EPA guidelines do not consider macropollutants $\left(\mathrm{NO}_{2}, \mathrm{PM}, \mathrm{O}_{3}\right.$ and carbon monoxide), the risk related to the exposure of the individuals to macropollutants can be anyway estimated through the several cohort studies available in the literature, which allow obtaining the so-called relative risk $(R R)$, i.e., the incremental probability that an effect occurs in an exposed population with respect to the probability that the same effect occurs in an unexposed one [23]. The literature provides $R R$ values concerning the incidence of specific non-cancer pathologies that require hospitalisation (e.g., respiratory and cardiovascular diseases) and the incidence of mortality. $R R$ values are inserted in parametrised doseresponse functions of the following type: 


$$
\Delta y=-P \cdot y_{0} \cdot\left(e^{-R R \cdot \Delta C}-1\right),
$$

where $\Delta y$ is the excess annual incidence of a specific effect, $P$ is population exposed to the increase of the annual mean concentration of a specific air pollutant $(\triangle C)$ with respect to the unexposed population and $y_{0}$ is the baseline prevalence of the effect per year [24]. $\Delta C$ and $R R$ are normally expressed as $\mu \mathrm{g} / \mathrm{m}^{3}$ and its reciprocal, respectively. Values of $y_{0}$ may be obtained either from the same cohort studies reporting the $R R$ values chosen or, if available, from national or international statistics database. The more specific the statistics are for the local population, the higher is the expected accuracy of the results.

\subsection{Verification of the exposure acceptability}

In the case of cancer risk, no threshold values are considered below which the impact can be considered as null, since a single damaged cell might be sufficient to initiate the development of cancer. However, the cancer risk may be considered as acceptable if comprised between $10^{-6}$ and $10^{-4}$ [25]. In the Italian legislation, $10^{-6}$ is the acceptable cancer risk related to the emission of a single pollutant from a single source, while this value is increased to $10^{-5}$ when considering more pollutants from a single source [26]. Considered the purpose of the present methodology, $10^{-5}$ is selected as the reference value to assess the acceptability of the overall cancer risk in the area, and the consequent possibility to start new industrial activities. This value is precautionary, since the methodology considers the as wider as possible number of emission sources in the area. Concerning the non-carcinogenic risk, the doses of each contaminant are divided by a contaminant-specific reference dose established by the US-EPA [21], below which no effects occur. This ratio is the so-called hazard index (HI). $\mathrm{HI}<1$ indicates the absence of toxic non-cancer impacts for health.

To quantify the impacts related to the inhalation exposure to macropollutants, the following procedure can be applied:

- convert the population density vector map into a raster map reporting the number of inhabitants in each cell (the cell size should equal that used in the dispersion model);

- for each pollutant and for the corresponding effects of interest, apply the dose-response function to each cell, now characterised by the pollutant concentration and the number of inhabitants; depending on the effects, it could be necessary to use the maximum concentrations instead of the annul mean ones;

- $\quad$ cell by cell and for each pollutant, quantify the effects in terms of hospitalisation for different health outcomes and of mortality;

- calculate the total number of cases within the computational domain;

- estimate the economic costs for the society, for instance through the cost functions defined by the ExternE methodology [27].

By applying the population raster map to the cancer risk map, it is possible to estimate the potential annual cancer cases related to air pollution within the target area. In this case, the economic quantification of the impacts is not feasible, due to the variability of the course of the disease among individuals.

To evaluate the incidence of one or more additional emission sources in the target area, the same methodology must be reapplied by replacing the current emission sources with the additional ones. By adding up the risk maps related to the current situations and the corresponding risk maps related to the additional sources, the new risk maps will take into account the contribution of the additional emission sources. If the new scenario did not comply with one of the acceptability criteria, or if the additional sources implied unacceptable 
sanitary impacts and social costs (according to criteria to be defined with the local authorities), it would be necessary to consider strategies to compensate the impacts or, eventually, deny the authorisation to the new activities.

\subsection{Evaluation of compensation measures}

To evaluate compensation strategies, it is necessary to define alternative emissive scenarios and apply again the methodology on the dominant emission sources of the area. Once the health risk will have been estimated depending on the air pollutants (macropollutants, carcinogenic micropollutants or toxic non-carcinogenic micropollutants), the acceptability of the risk must be verified.

\section{CRITICAL ASPECTS}

The methodology presented in Section 2 follows a multi-step approach that makes use of several procedures. Generally, at present, the latter are poorly harmonised and require a huge amount of input data, both in terms of quantity and in terms of variety. Therefore, efforts are necessary to facilitate the retrieving of the data of interest and to make them compatible with the procedures adopted. A set of tools could be developed by the scientific community to facilitate the fast conversion of data into the format required by who applies the methodology. However, the public sector (e.g., environmental agencies) should ease the operation of data collection by making all the data of interest available to the public and reachable on a dedicated web portal. In addition, the following critical aspects are worth consideration:

1. the background contributions of heavy metals, dioxin, benzene, polycyclic aromatic hydrocarbons and carbon monoxide would be neglected in the assessment of the health impacts if those pollutants were not measured by the air quality monitoring stations located in the target area;

2. at present, an institutional methodology to estimate the transfer of toxic and persistent pollutants from the environment to animals and from animals to meat, dairy products and eggs is still absent;

3. the different databases required to extract relevant emission factors might consider different pollutants and, in some cases, pollutants that are aggregated in different manners.

The first point would require specific monitoring campaigns covering the unmonitored pollutants. However, two assumptions could be formulated on this aspect:

- the background concentrations of benzene and carbon monoxide, whose presence is generally limited to the proximity with emission sources [28], [29], might be neglected;

- the possible underestimation of persistent pollutants might be compensated by the precautionary strategy of considering that the diet is $100 \%$ based on locally-grown food.

The second point implies that, in case farm animals present in the target area, the estimation of the health impacts should consider animal- and pollutant-specific food-chain models available in the literature. As an example, McLachlan [30] developed a model to assess the accumulation of dioxins and furans through the feed-cattle-beef/milk chain.

The third point entails the need for harmonising different databases, introducing hypotheses or neglecting some pollutants.

In the light of the previous considerations, the role of public institutions is crucial in view of a homogenisation of data and procedures that would avoid wrong conclusions in a health impact assessment or approximations that might underestimate the real impacts. 


\section{CONCLUSIONS}

The present paper proposes a methodology aimed at assessing the health risk related to the air quality in a target area and a criterion to evaluate the acceptability of the additional incidence of a new emissive activity. The criterion is based on the concepts of acceptable cancer risk, unitary $\mathrm{HI}$ and the compliance with local sanitary and economic targets, respectively considering the effects of carcinogenic micropollutants, the toxic noncarcinogenic effects of micropollutants and macropollutants. The methodology actually consists in a sequence of consolidated procedures (emission calculation, dispersion modelling, health risk estimation, external cost estimation) and novel steps (compensation criterion), which were here presented in a unified manner, in order to serve as guidelines to local administrations. The implementation of this methodology could facilitate the decisionmaking process concerning the authorisation procedure of new industrial/civil activities, but requires efforts by the public sector at local and national/international levels. Specifically, additional monitoring campaigns in the areas under investigation might be necessary to retrieve background contributions of air pollutants that are not monitored by the air quality stations in the target areas. In addition, at a national/international level, the public sector should support the harmonisation of different sources of data to facilitate the application of the complete methodology presented here.

\section{ACKNOWLEDGEMENT}

The authors wish to thank the ATS Consortium for the financial support to the research that was the starting point of this article.

\section{REFERENCES}

[1] UN Environmental Assembly, Air. http://web.unep.org/environmentassembly/air. Accessed on: 30 Jan. 2019.

[2] De Marco, A. et al., Impacts of air pollution on human and ecosystem health, and implications for the National Emission Ceilings Directive: Insights from Italy. Environment International, 125, pp. 320-333, 2019.

[3] Schiavon, M., Ragazzi, M., Rada, E.C., Magaril, E. \& Torretta, V., Towards the sustainable management of air quality and human exposure: Exemplary case studies. WIT Transactions on Ecology and the Environment, vol. 230, WIT Press: Southampton and Boston, pp. 489-500, 2018.

[4] Rada, E.C., The sustainable city and air pollution. WIT Transactions on Ecology and the Environment, vol. 191, WIT Press: Southampton and Boston, pp. 1369-1380, 2014.

[5] European Environment Agency, Air Quality in Europe - 2018 Report. www.eea.europa.eu//publications/air-quality-in-europe-2018. Accessed on: 30 Jan. 2019.

[6] Tian, Y., Yao, X. \& Chen, L., Analysis of spatial and seasonal distributions of air pollutants by incorporating urban morphological characteristics. Computers, Environment and Urban Systems, 75, pp. 35-48, 2019.

[7] Cai, J. et al., Association between airborne fine particulate matter and residents' cardiovascular diseases, ischemic heart disease and cerebral vascular disease mortality in areas with lighter air pollution in China. International Journal of Environmental Research and Public Health, 15(9), p. 1918, 2018.

[8] Murakami, K., Itsubo, N., Kuriyama, K., Yoshida, K. \& Tokimatsu, K., Development of weighting factors for G20 countries. Part 2: Estimation of willingness to pay and 
annual global damage cost. International Journal of Life Cycle Assessment, 23(12), pp. 2349-2364, 2018.

[9] Pimpin, L. et al., Estimating the costs of air pollution to the National Health Service and social care: An assessment and forecast up to 2035. PLoS Medicine, 15(7), e1002602, 2018.

[10] Schiavon, M. et al., Assessing the air quality impact of nitrogen oxides and benzene from road traffic and domestic heating and the associated cancer risk in an urban area of Verona (Italy). Atmospheric Environment, 120, pp. 234-243, 2015.

[11] Swab, C., Allen, P., Armitage, S. \& Biberic, A., 2014 residential wood combustion survey: Results overview and spatial allocation of emissions estimates. Atmospheric Environment, 198, pp. 12-22, 2019.

[12] Ionescu, G., Apostol, T., Rada, E.C., Ragazzi, M. \& Torretta, V., Critical analysis of strategies for PM reduction in urban areas. UPB Scientific Bulletin, Series D: Mechanical Engineering, 75, pp. 175-186, 2013.

[13] Rodrigues, S.M. \& Römkens, P.F.A.M., Human health risks and soil pollution. Soil Pollution, eds A.C. Duarte, A. Cachada \& T. Rocha-Santos, Elsevier Academic Press: London, pp. 217-250, 2018.

[14] Copernicus Land Monitoring Service, CLC 2012. http://and.copernicus.eu/paneuropean/corine-land-cover/clc-2012/view. Accessed on: 28 Jan. 2019.

[15] European Environment Agency, EMEP/EEA air pollutant emission inventory guidebook 2016. www.eea.europa.eu/publications/emep-eea-guidebook-2016. Accessed on: 23 Jan. 2019.

[16] EMISIA, COPERT 5 - Introduction. https://copert.emisia.com/. Accessed on: 23 Jan. 2019.

[17] Tomasi, E., Antonacci, G., Giovannini, L., Zardi, D. \& Ragazzi, M., Atmospheric dispersion modelling with AERMOD for comparative impact assessment of different pollutant emission sources in an Alpine valley. WIT Transactions on Ecology and the Environment, vol. 198, WIT Press: Southampton and Boston, pp. 431-442, 2015.

[18] INEMAR, XWiki. www.inemar.eu/xwiki/bin/view/Inemar/WebHome. Accessed on: 24 Jan. 2019.

[19] Wackernagel, H., Multivariate Geostatistics, Springer: Berlin, Heidelberg, pp. 74-81, 1995.

[20] U.S. Environmental Protection Agency, Office of Solid Waste and Emergency Response, Human health risk assessment protocol for hazardous waste combustion facilities (final), Report no. EPA530-R-05-006.

[21] U.S. Environmental Protection Agency, Integrated risk information system (IRIS). www.epa.gov/IRIS/. Accessed on: 20 Dec. 2018.

[22] INRAN, National investigation on food consumption in Italy. http://nut.entecra.it/710/I_consumi_alimentari_INRAN-SCAI_2005-06.html. Accessed on: 17 Jan. 2019. (In Italian.)

[23] National Cancer Institute, Definition of relative risk. www.cancer.gov/publications/ dictionaries/cancer-terms/def/relative-risk. Accessed on: 30 Jan. 2019.

[24] Logue, J.M., Price, P.N., Sherman, M.H. \& Singer, B.C., A method to estimate the chronic health impact of air pollutants in U.S. residences. Environmental Health Perspectives, 120(2), pp. 216-222, 2012.

[25] U.S. Environmental Protection Agency, Residual risk report to congress. www.epa.gov/sites/production/files/2013-08/documents/risk_rep.pdf. Accessed on: 23 Jan. 2019. 
[26] Italian Republic, Decree 4/2008 - Additional corrective and integrative measures to the decree 152/2006 on environmental legislation. Official Journal of the Italian Republic, 24, 2008.

[27] EMRC, Cost-benefit analysis of final policy scenarios for the EU clean air package. http://ec.europa.eu/environment/air/pdf/TSAP\%20CBA.pdf. Accessed on: 24 Jan. 2019.

[28] Skorokhod, A.I., Berezina, E.V., Moiseenko, K.B., Elansky, N.F. \& Belikov, I.B., Benzene and toluene in the surface air of northern Eurasia from TROICA-12 campaign along the Trans-Siberian Railway. Atmospheric Chemistry and Physics, 17, pp. 55015514, 2017.

[29] Zhang, K. \& Batterman, S., Near-road air pollutant concentrations of CO and $\mathrm{PM}_{2.5}$ : A comparison of MOBILE6.2/CALINE4 and generalized additive models. Atmospheric Environment, 44, pp. 1740-1748, 2010.

[30] McLachlan, M.S., A simple model to predict accumulation of PCDD/Fs in an agricultural food chain. Chemosphere, 34, pp. 1263-1276, 1997. 